\title{
A novel and dominant factor that mediates oxidative stress-induced apoptotic signaling - autocrine/ paracrine mechanism of the secreted form of eukaryotic translation initiation factor $5 \mathrm{~A}$
}

\author{
Yoshinori Seko \\ Department of Biofunctional Microbiota, Graduate School of Medicine, Juntendo University, Bunkyo-ku, Tokyo 113-8421, Japan.
}

Correspondence to: Dr. Yoshinori Seko, Department of Biofunctional Microbiota, Graduate School of Medicine, Juntendo University, Bunkyo-ku, Tokyo 113-8421, Japan. E-mail: sekoyosh-tky@umin.ac.jp

How to cite this article: Seko Y. A novel and dominant factor that mediates oxidative stress-induced apoptotic signaling autocrine/paracrine mechanism of the secreted form of eukaryotic translation initiation factor 5A. Vesse/ P/us 2020;4:22. http://dx.doi.org/10.20517/2574-1209.2020.14

Received: 6 May 2020 First Decision: 29 Jun 2020 Revised: 7 Jul 2020 Accepted: 15 Jul 2020 Published: 26 Jul 2020

Academic Editor: Cristina Vassalle Copy Editor: Cai-Hong Wang Production Editor: Jing Yu

\begin{abstract}
Oxidative stress plays a critical role in the pathogenesis of various disorders including cardiovascular diseases, such as ischemia/reperfusion (I/R) injury, atherosclerosis, dyslipidemia, chronic kidney disease (CKD), arrhythmia, and diabetic cardiovascular complications. Although reactive oxygen species (ROS) have been proposed as the key mediator of oxidative stress-induced cell injury, antioxidant therapies have failed in clinical trials, raising the possibility that some unknown mechanism other than ROS may be involved. In 2015, we reported a novel apoptosis-inducing humoral factor in conditioned medium from cardiac myocytes subjected to hypoxia/ reoxygenation. This novel 69th tyrosine-sulfated eukaryotic translation initiation factor 5A (elF5A) was rapidly secreted from cells in response to oxidative stress and then acted as an apoptosis-inducing ligand in an autocrine fashion. We termed the novel secreted form of elF5A "Oxidative stress-Responsive Apoptosis-Inducing Protein" (ORAIP). Evidence has accumulated that ORAIP may be a common and dominant apoptosis-inducer among various cell types in response to different types of oxidative stress and is involved in a wide spectrum of acute and chronic disorders. Among them, here, I summarize knowledge regarding the possible roles of ORAIP in myocardial and cerebral I/R injury, dyslipidemia in terms of atherosclerosis, cardiovascular complications in diabetes mellitus, and CKD.
\end{abstract}

Keywords: Atherosclerosis, chronic kidney disease, diabetes mellitus, eukaryotic translation initiation factor 5A,

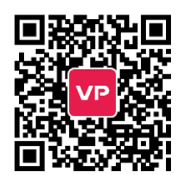


ischemia/reperfusion injury, oxidative stress, oxidative stress-responsive apoptosis inducing protein, reactive oxygen species

\section{INTRODUCTION}

Oxidative stress has been strongly implicated in the pathogenesis of various disorders including cardiovascular diseases, such as arrhythmia, heart failure, dyslipidemia, atherosclerosis, chronic kidney disease (CKD), diabetic cardiovascular complications, and in particular ischemia/reperfusion (I/R) injury. Oxidative stress induces reactive oxygen species (ROS) production, lipid peroxidation, protein oxidation, and DNA damage in the cells that lead to apoptosis. Until recently, ROS were proposed as the key mediator of oxidative stress-induced cell injury ${ }^{[1-3]}$. However, large scale antioxidants (including vitamins, free radical scavengers) clinical trials have been unsuccessful to improve the outcome of cardiovascular and cerebrovascular diseases in humans ${ }^{[4,5]}$, raising the possibility that there might be some unknown mechanism other than ROS that mediates oxidative stress-induced cell injury.

In 2015, we reported a novel apoptosis-inducing humoral factor in conditioned medium from cardiac myocytes subjected to hypoxia/reoxygenation. We reported that this novel secreted form of eukaryotic translation initiation factor 5A (eIF5A) was sulfated at the 69th tyrosine residue and contained more of the hypusinated isoform than the conventional cytosolic form of eIF5 $\mathrm{A}^{[6]}$. We found that eIF5A undergoes tyrosine-sulfation in the trans-Golgi and is rapidly secreted from cardiac myocytes in response to hypoxia/ reoxygenation. It then induces apoptosis by acting as a pro-apoptotic ligand in an autocrine fashion [Figure 1]. We termed this novel tyrosine-sulfated secreted form of eIF5A, Oxidative stress-Responsive ApoptosisInducing Protein (ORAIP ${ }^{[6]}$. eIF5A, a member of eIFs regulating the translation initiation step of protein synthesis, is the only known protein to contain the unique amino acid hypusine, which is formed posttranslationally via a two-step enzymatic reaction with deoxyhypusine synthase (DHS) and deoxyhypusine hydroxylase [Figure 1$]^{[7]}$. eIF5A is primarily localized to the cytoplasm, where hypusinated eIF5A facilitates the translation of mRNAs that are involved in cell proliferation. We found that myocardial I/R (but not ischemia alone) rapidly and markedly increased plasma levels of ORAIP, which returned to the control level within $60 \mathrm{~min}$. In vivo treatment with an anti-ORAIP neutralizing monoclonal antibody (mAb) significantly reduced myocardial I/R injury ${ }^{[6]}$. It seems that secretion of ORAIP is specific to oxidative stresses including I/R, hypoxia/reoxygenation, ultraviolet light, ionizing radiation, cold/warm-stress (heat shock), and blood acidification ${ }^{[8]}$, then plays a crucial role in inducing apoptosis of target cells such as cardiac and skeletal myocytes, neurons, and cancer cells. Especially, these cells need substantial amounts of oxygen for their activities, making them very sensitive to oxygen concentrations and hence susceptible to oxidative stress-induced apoptosis mediated by ORAIP.

These results strongly suggested that ORAIP may be a specific biomarker and critical therapeutic target for oxidative stress-induced cell injury. We also found that the plasma levels of ORAIP were markedly elevated in patients with chronic disorders such as CKD, atrial fibrillation, heart failure, dyslipidemia, diabetes mellitus (DM), and diabetic retinopathy, in which oxidative stress plays a critical role in the pathogenesis ${ }^{[9-12]}$. Thus, evidence has accumulated that ORAIP may be a common and dominant apoptosisinducing ligand among various cell types in response to different types of oxidative stresses involved in a wide spectrum of acute and chronic disorders, especially cardiovascular diseases.

\section{MYOCARDIAL AND CEREBRAL I/R INJURY}

It has been long believed that neutrophils infiltrate into myocardial tissues subjected to $\mathrm{I} / \mathrm{R}$ and cause myocardial damage, known as reperfusion injury, due to progressive capillary plugging by neutrophils that cause capillary no-reflow as well as ROS formation ${ }^{[13-15]}$. Because reperfusion-induced apoptotic cell death 


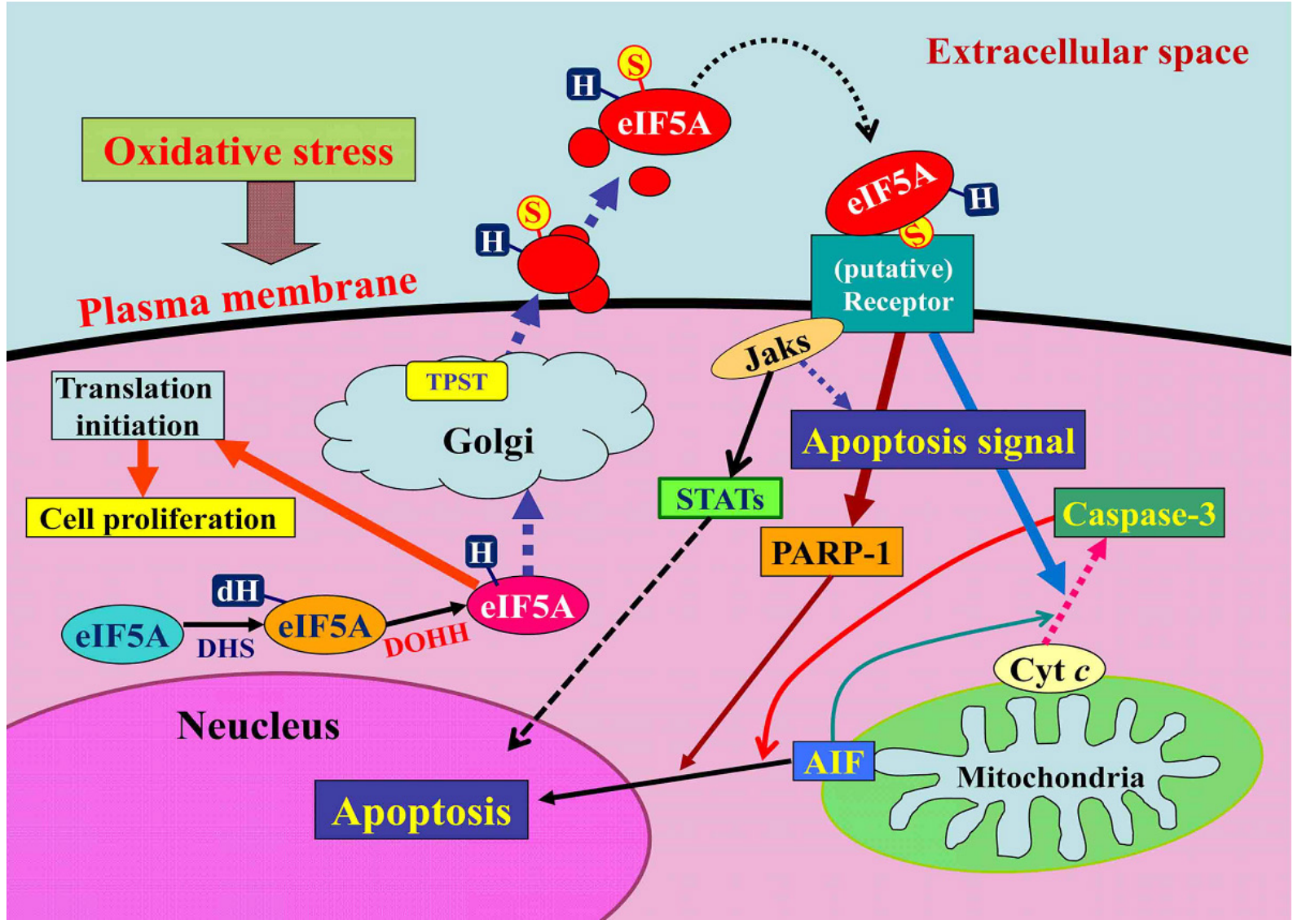

Figure 1. A model for the mechanism by which oxidative stress induces apoptosis via the autocrine secretion of elF5A (oxidative stressresponsive apoptosis-inducing protein ${ }^{[6]}$. AIF: apoptosis-inducing factor; cyt c: cytochrome c; $\mathrm{dH}$ : deoxyhypusine; DHS: deoxyhypusine synthase; DOHH: deoxyhypusine hydroxylase; H: hypusine; Jaks: Janus kinases; S, sulfated; PARP-1: poly (ADP-ribose) polymerase-1; STATs: signal transducers and activators of transcriptions; TPST: tyrosyl protein sulfotransferase; elF5A: eukaryotic translation initiation factor $5 \mathrm{~A}$

cannot be prevented by neutrophil depletion, some mechanism other than neutrophil infiltration triggered by reperfusion may mediate apoptotic signaling before neutrophil infiltration occurs ${ }^{[16,17]}$. To exclude the effects of neutrophil infiltration, using an in vitro model of myocardial I/R, we identified a novel apoptosis-inducing humoral factor in conditioned medium from cardiac myocytes subjected to hypoxia/ reoxygenation, that is ORAIP ${ }^{[6]}$. Myocardial or cerebral I/R rapidly and markedly increased ORAIP levels in plasma and cerebrospinal fluid, whereas ischemia alone did not alter ORAIP levels ${ }^{[6,18]}$. In vivo treatment with the anti-ORAIP neutralizing mAb dominantly reduced myocardial or cerebral I/R injury as compared with conventional therapies ${ }^{[6,18]}$. This suggests that ORAIP plays a pivotal role in I/R-induced tissue injury and can be an oxidative stress-specific biomarker.

Muscle cells (especially cardiac myocytes) and cerebral neurons demand a lot of oxygen for their activities, making them very sensitive to oxygen concentrations and hence susceptible to oxidative stress, such as I/R injury, in which ORAIP plays a major role. It is thought that patients with significant stenosis of coronary or cerebral arteries are often subjected to silent myocardial or cerebral I/R, even subacute myocardial or cerebral infarction, which may lead to accumulation of cell injury, resulting in ischemic cardiomyopathy or lacunar infarction. Therefore, anti-ORAIP therapy may be effective in patients with stable ischemic heart or cerebrovascular diseases, as well as those subjected to reperfusion therapy for acute myocardial and cerebral infarction. 


\section{DYSLIPIDEMIA AND ATHEROSCLEROSIS}

Oxidative stress plays an important role in the pathogenesis of dyslipidemia. Like other risk factors for atherosclerosis, such as DM, hypertension, and smoking, it is believed to play a critical role in atherosclerotic plaque formation and rupture ${ }^{[19]}$. According to the low-density lipoprotein cholesterol (LDL-C) modification hypothesis of atherogenesis, normal plasma LDL-C is modified by oxidative stress in the arterial wall into oxidized LDL (oxLDL), then bound and taken up through scavenger receptors by monocytes/macrophages chemoattracted by the oxLDL in the arterial wall. Progressive accumulation of cholesterol in the monocytes/macrophages turns them into foam cells ${ }^{[20-22]}$. Then, the accumulation of foam cells in the arterial lesions leads to atherosclerotic plaque formation. Furthermore, it has been postulated that plaque rupture is often associated with thrombosis and plays a major role in acute coronary syndrome and cerebral infarction. However, the precise mechanism of plaque vulnerability leading to rupture has been unclear.

To investigate whether ORAIP has a role in atherosclerosis, especially in the mechanism of plaque rupture, we analyzed plasma levels of ORAIP and oxLDL in patients with dyslipidemia as well as heterozygous familial hypercholesterolemia (HeFH). We also examined the expression of ORAIP and the levels of oxLDL in atherosclerotic coronary arterial tissues obtained from HeFH patients with a coronary artery bypass graft. Plasma levels of LDL-C, oxLDL, and ORAIP in HeFH were significantly elevated as compared with those in dyslipidemia. ORAIP and oxLDL colocalized in the plaque lesion of coronary arteries from a HeFH patient [unpublished observation]. These findings suggested that high levels of plasma LDL-C facilitate oxidative stress in the arterial wall which, in turn, induces oxLDL accumulation, plaque formation, and ORAIP secretion, resulting in arterial cell apoptosis leading to plaque rupture. Although further investigations are needed, our findings suggest that anti-ORAIP therapy could be a way to reduce atherosclerotic plaque rupture and cardiovascular injury in patients with dyslipidemia, especially $\mathrm{HeFH}$.

\section{DIABETIC CARDIOVASCULAR COMPLICATIONS}

Diabetic cardiovascular complications include microangiopathy, atherosclerotic macroangiopathy, and muscle injury. There are four main molecular mechanisms implicated in the hyperglycemia-induced cell injury: increased polyol pathway flux, increased advanced glycation end-product formation, activation of protein kinase $\mathrm{C}$ isoforms, and increased hexosamine pathway flux. All of these mechanisms have been proposed to reflect the hyperglycemia-induced overproduction of ROS by the mitochondria ${ }^{[23]}$. We reported previously ${ }^{[24]}$ that plasma ORAIP levels in DM model rats were markedly elevated during the diabetic phase as compared to the non-diabetic control phase, and that there was a significant positive correlation between plasma levels of glucose and ORAIP. We also found that high glucose-induced massive apoptosis of cultured cardiac myocytes, which was largely suppressed by neutralizing anti-ORAIP mAbs in vitro. Furthermore, recombinant-ORAIP induced the apoptosis of pancreatic ß-cells in vitro. Hyperglycemia induces pancreatic $B$-cell apoptosis leading to insulin deficiency ${ }^{[25]}$. These findings strongly suggest that ORAIP plays a pivotal role in hyperglycemia-induced myocardial injury as well as pancreatic ß-cell injury in DM. ORAIP may be a biomarker and a critical therapeutic target for myocardial injury and progression of insulin deficiency due to pancreatic ß-cell injury in patients with DM.

\section{Microangiopathy}

Diabetic microangiopathy is often associated with three major complications: nephropathy, retinopathy, and neuropathy. It is proposed that capillary endothelial cells and glomerular mesangial cells are preferentially injured by hyperglycemia because these cells cannot reduce the transport of glucose inside the cell when they are exposed to hyperglycemia ${ }^{[26]}$. Although we do not have data on whether these cells secrete ORAIP in response to hyperglycemia, as we reported previously that plasma levels of ORAIP were markedly elevated in patients undergoing dialysis largely due to diabetic nephropathy ${ }^{[9]}$. These elevated 
levels of ORAIP played a role in other complications such as cardiovascular injury and sarcopenia in these patients.

For diabetic retinopathy, it is thought that vascular endothelial growth factor (VEGF) plays a critical role in retinal neovascularization and diabetic macular edema. VEGF also plays a protective role against retinal apoptosis induced by oxidative stress (perhaps due to retinal I/R ${ }^{[26,27]}$. We reported that vitreous body concentrations of ORAIP were significantly increased in diabetic retinopathy, especially proliferative diabetic retinopathy, suggesting that ORAIP plays a role in oxidative stress-induced retinal cell injury ${ }^{[12]}$. Therefore, the combination of anti-VEGF and anti-ORAIP therapies will be complementary against ischemic as well as I/R injury in diabetic retinopathy.

Because neurons (especially cerebral neurons) are susceptible to oxidative stress-induced cell injury, the long-term elevation of plasma ORAIP levels induced by hyperglycemia should injure peripheral neurons as well.

\section{Atherosclerotic macroangiopathy}

$\mathrm{DM}$ is often associated with hypertriglyceridemia due to insulin resistance. In addition, hyperglycemia in DM induces oxidative stress, which facilitates LDL-C oxidation in arterial tissues leading to plaque formation. Thus, hyperglycemia in DM often causes dyslipidemia, resulting in the development of atherosclerotic macroangiopathy involving coronary, cerebral, and peripheral arteries. Atherosclerosis in these large arteries leads to life-threatening ischemic heart disease, cerebral infarction, and arteriosclerosis obliterans. As mentioned in the "Dyslipidemia and atherosclerosis" section, oxidative stress in arterial tissues induces ORAIP secretion, which may result in apoptosis of arterial cells leading to plaque rupture. Further investigations are needed to prove the usefulness of anti-ORAIP therapy with a neutralizing $\mathrm{mAb}$ in protecting from atherosclerotic plaque rupture and cardiovascular injury in patients with DM as well as dyslipidemia.

\section{Muscle injury}

Myocardial injury, as well as sarcopenia, often develops later in the course of DM, causing various types of arrhythmias (such as atrial fibrillation and paroxysmal supraventricular tachycardia) and heart failure (such as diabetic cardiomyopathy). Because cardiac and skeletal myocytes are susceptible to oxidative stressinduced apoptosis mediated by ORAIP, and ORAIP has a critical role in hyperglycemia-induced myocardial injury $^{[24]}$, hyperglycemia-induced high plasma levels of ORAIP may also mediate the development of sarcopenia.

Taken together, these data offer a possible anti-ORAIP therapy against vascular as well as cardiac and skeletal muscle injury involved in DM.

\section{CARDIOVASCULAR COMPLICATIONS IN CKD}

Cardiovascular complications critically affect the morbidity and mortality of CKD, especially in endstage renal disease patients on dialysis. Because conventional cardiovascular risk factors such as DM, hypertension, hypercholesterolemia, and smoking are often associated with CKD, oxidative stress has been implicated in the mechanism of cardiovascular injury in $\mathrm{CKD}^{[28]}$, though the precise mechanism is still unclear. To investigate the roles of ORAIP in oxidative stress-induced cardiovascular injury in CKD, we analyzed the plasma levels of ORAIP in patients with the end-stage renal disease just before and after dialysis $^{\left[{ }^{[\rho]}\right.}$. Plasma ORAIP levels before dialysis were markedly elevated $[93.6 \pm 5.1$ (mean $\pm \mathrm{SE}$ ) ng/mL] as compared with those of control subjects $(6.6 \pm 1.5 \mathrm{ng} / \mathrm{mL})$. After dialysis, plasma levels of ORAIP were not decreased, but rather slightly (and significantly) increased to $98.5 \pm 5.7 \mathrm{ng} / \mathrm{mL}(P=0.0122)$, suggesting that ORAIP may be a little concentrated, but not eliminated by dialysis. 
To assess the effects of high concentrations of plasma ORAIP on the cardiovascular injury, we analyzed plasma levels of cardiac troponin T and brain natriuretic peptide (BNP). Plasma levels of cardiac troponin $\mathrm{T}$ were considerably elevated and there was a tendency of a positive correlation (but not significant) between plasma levels of ORAIP and cardiac troponin $\mathrm{T}^{[\rho]}$. Because many factors may contribute to myocardial injury, the absence of a significant positive correlation between plasma levels of ORAIP and cardiac troponin $\mathrm{T}$ does not exclude the possibility that ORAIP may contribute to the myocardial injury. Plasma BNP levels were markedly elevated. There was also a tendency of a positive correlation (but not significant and weaker than that for cardiac troponin T) between plasma levels of ORAIP and BNP. This seems reasonable because cardiac troponin $\mathrm{T}$ directly reflects myocardial injury whereas BNP can be affected by several other factors such as overhydration in patients with end-stage renal disease on dialysis as well as myocardial injury. Although the primary mechanism of oxidative stress generation in CKD is unclear, the elevated plasma levels of ORAIP may cause sarcopenia and renal microvascular injury, resulting in the progression of CKD. Although further in vivo treatment studies are needed, these data offer support for a possible anti-ORAIP therapy to at least partially protect from cardiovascular injury and sarcopenia in patients with CKD.

\section{CONCLUSION}

For life, oxygen is a double-edged sword because it is not only essential for vital activities, but excessive oxygen can be harmful to various cells, known as oxidative stress. Since we discovered a novel secreted form of eIF5A to be ORAIP, we found that plasma levels of ORAIP were elevated in a wide range of acute and chronic disorders (including cardiovascular diseases), in which oxidative stress is known to be involved in the pathogenesis. Using animal models of such disorders, we also demonstrated that anti-ORAIP therapy with neutralizing mAbs could critically reduce oxidative stress-induced cell injury. We speculate that excessive external stresses involved in various pathological conditions including electromagnetic waves (such as ionizing radiation and ultraviolet), physicochemical stimuli (such as heat-shock, acidification, pressure overload, and high osmotic pressure) as well as excessive oxygen (such as ischemia/reperfusion) may trigger the oxidative stress-sensing mechanism of the cells, which results in the secretion of ORAIP. Then, secreted ORAIP induces apoptosis of the cells in an autocrine/paracrine fashion, that contributes to the progression of these disorders. Recently, we identified a cell-surface receptor for ORAIP (that is, ORAIP-receptor; unpublished data), through which ORAIP transduces the apoptotic signal intracellularly. From data of hyperglycemia-induced cell apoptosis ${ }^{[24]}$, it is strongly suggested that the ORAIP/ORAIPreceptor pathway mediates a great part of oxidative stress-induced cell injury. Therefore, anti-ORAIP therapy may be very efficient and effective in combination with conventional therapies against oxidative stress-mediated cell injury in various disorders, especially cardiovascular diseases.

\section{DECLARATIONS}

\section{Authors' contributions}

Seko Y contributed solely to the article.

\section{Availability of data and materials}

Not applicable.

\section{Financial support and sponsorship}

None.

\section{Conflicts of interest}

The author declared that there are no conflicts of interest. 


\section{Ethical approval and consent to participate}

Not applicable.

\section{Consent for publication}

Not applicable.

\section{Copyright}

(c) The Author(s) 2020.

\section{REFERENCES}

1. Chien CT, Lee PH, Chen CF, Ma MC, Lai MK, et al. De novo demonstration and co-localization of free-radical production and apoptosis formation in rat kidney subjected to ischemia/reperfusion. J Am Soc Nephrol 2001;12:973-82.

2. Kaminski KA, Bonda TA, Korecki J, Musial WJ. Oxidative stress and neutrophil activation - the two keystones of ischemia/reperfusion injury. Int J Cardiol 2002;86:41-59.

3. Madamanchi NR, Vendrov A, Runge MS. Oxidative stress and vascular disease. Arterioscler Thromb Vasc Biol 2005;25:29-38.

4. Vogiatzi G, Tousoulis D, Stefanadis C. The role of oxidative stress in atherosclerosis. Hellenic J Cardiol 2009;50:402-9.

5. Myung SK, Ju W, Cho B, Oh SW, Park SM, et al. Efficacy of vitamin and antioxidant supplements in prevention of cardiovascular disease: systematic review and meta-analysis of randomised controlled trials. Br Med J 2013;346:f10.

6. Seko Y, Fujimura T, Yao T, Taka H, Mineki R, et al. Secreted tyrosine sulfated-eIF5A mediates oxidative stress-induced apoptosis. Sci Rep 2015;5:13737.

7. Caraglia M, Marra M, Giuberti G, D’Alessandro AM, Budillon A, et al. The role of eukaryotic initiation factor 5A in the control of cell proliferation and apoptosis. Amino Acids 2001;20:91-104.

8. Yao T, Fujimura T, Murayama K, Seko Y. Plasma levels of oxidative stress-responsive apoptosis inducing protein (oraip) in rats subjected to physicochemical oxidative stresses. Bioscience Rep 2016;36: e00317.

9. Tanaka K, Yao T, Fujimura T, Murayama K, Fukuda S, et al. Marked elevation of plasma levels of oxidative stress-responsive apoptosis inducing protein in dialysis patients. Kidney Int Rep 2016;1:321-4.

10. Yao T, Tanaka K, Fujimura T, Murayama K, Fukuda S, et al. Plasma levels of oxidative stress-responsive apoptosis inducing protein (ORAIP) in patients with atrial fibrillation. Int J Cardiol 2016;222:528-30.

11. Tanaka K, Yao T, Sato K, Okumura K, Seko Y. Oxidative stress-responsive apoptosis inducing protein (ORAIP) plays a critical role in the cardiac injury in patients with heart failure. Ann Pharmacol Pharm 2017;2:1100.

12. Suzuki Y, Yao T, Okumura K, Seko Y, Kitano S. Elevation of vitreous body concentrations of oxidative stress-responsive apoptosis inducing protein (ORAIP) in diabetic retinopathy. Graefes Arch Clin Exp Ophthalmol 2019;257:1519-25.

13. Schmid-Schonbein GW. Capillary plugging by granulocytes and the no-reflow phenomenon in the microcirculation. FASEB J 1987;46:2397-401.

14. Mehta JL, Nichols WW, Mehta P. Neutrophils as potential participants in acute myocardial ischemia: relevance to reperfusion. J Am Coll Cardiol 1988;11:1309-16.

15. Simpson PJ, Lucchesi BR. Free radicals and myocardial ischemia and reperfusion injury. J Lab Clin Med 1987;110:13-30.

16. Gottlieb RA, Burleson KO, Kloner RA, Babior BM, Engler RL. Reperfision injury induces apoptosis in rabbit cardiomyocytes. J Clin Invest 1994;94:1621-8.

17. Bielawska AE, Shapiro JP, Jiang L, Melkonyan HS, Piot C, et al. Ceramide is involved in triggering of cardiomyocytes apoptosis induced by ischemia and reperfusion. Am J Pathol 1997;151:1257-63.

18. Kishimoto M, Suenaga J, Takase H, Araki K, Yao T, et al. Oxidative stress-responsive apoptosis inducing protein (ORAIP) plays a critical role in cerebral ischemia/reperfusion injury. Sci Rep 2019;9:13512.

19. Bonomini F, Tengattini S, Fabiano A, Bianchi R, Rezzani R. Atherosclerosis and oxidative stress. Histol Histopathol 2008;23:381-90.

20. Morel DW, Hessler JR, Chisolm GM. Low density lipoprotein cytotoxicity induced by free radical peroxidation of lipid. J Lipid Res $1983 ; 24: 1070-6$.

21. Steinbrecher UP, Parthasarathy S, Leake DS, Witztum JL, Steinberg D. Modification of low density lipoprotein by endothelial cells involves lipid peroxidation and degradation of low density lipoprotein phospholipids. Proc Natl Acad Sci USA 1984;81:3883-7.

22. Steinberg D, Parthasarathy S, Carew TE, Khoo JC, Witztum JL. Beyond cholesterol. Modifications of low-density lipoprotein that increase its atherogenicity. N Engl J Med 1989;320:915-24.

23. Brownlee M. Biochemistry and molecular cell biology of diabetic complications. Nature 2001;414:813-20.

24. Yao T, Fujimura T, Murayama K, Okumura K, Seko Y. Oxidative stress-responsive apoptosis inducing protein (ORAIP) plays a critical role in high glucose-induced apoptosis in rat cardiac myocytes and murine pancreatic $\beta$-cells. Cells 2017;6:35.

25. Tomita T. Apoptosis in pancreatic $\beta$-islet cells in Type 2 diabetes. Bosn J Basic Med Sci 2016;16:162-79.

26. Brownlee M. The pathophysiology of diabetic complications. Diabetes 2005;54:1615-25.

27. Amato R, Biagioni M, Cammalleri M, Dal Monte M, Casini G. VEGF as a survival factor in ex vivo models of early diabetic retinopathy. Invest Ophthalmol Vis Sci 2016;57:3066-76.

28. Locatelli F, Canaud B, Eckardt KU, Stenvinkel P, Wanner C, et al. Oxidative stress in end-stage renal disease: an emerging threat to patient outcome. Nephrol Dial Transplant 2003;18:1272-80. 